PROCEEDINGS OF THE

AMERICAN MATHEMATICAL SOCIETY

Volume 127, Number 7, Pages 2035-2040

S 0002-9939(99)04900-X

Article electronically published on February 18, 1999

\title{
A QUASISYMMETRIC SURFACE WITH NO RECTIFIABLE CURVES
}

\author{
CHRISTOPHER J. BISHOP
}

(Communicated by Frederick W. Gehring)

\begin{abstract}
There is a quasiconformal mapping $f$ of $\mathbb{R}^{3}$ to itself such that the image of $\mathbb{R}^{2} \times\{0\}$ contains no rectifiable curves.
\end{abstract}

\section{INTRODUCTION}

A mapping $f: X \rightarrow Y$ is called quasiconformal if there is an $H<\infty$ so that the linear dilatation

$$
H(x) \equiv \lim _{r \rightarrow 0} \frac{\max _{|x-y|=r}|f(x)-f(y)|}{\min _{|x-y|=r}|f(x)-f(y)|} \leq H,
$$

for every $x$. The purpose of this note is to prove

Theorem 1.1. There is a quasiconformal mapping $f$ of $\mathbb{R}^{3}$ to itself such that

$$
C^{-1} \leq \frac{|f(z)-f(w)|}{\varphi(|z-w|)} \leq C, \quad \forall z, w \in \mathbb{R}^{2} \times\{0\},
$$

for some $C<\infty$ and some increasing function $\varphi$ such that

$$
\lim _{t \rightarrow 0} \varphi(t) / t=\infty .
$$

For $E \in \mathbb{R}^{2} \times\{0\}$, if the image $f(E)$ can be covered by balls $\left\{B\left(f\left(x_{j}\right), \varphi\left(r_{j}\right)\right\}\right.$, then $E$ is covered by balls $B\left(x_{j}, C r_{j}\right)$. Then (2) easily implies that if $f(E)$ has finite $\alpha$-dimensional Hausdorff measure, then $E$ has zero $\alpha$-measure. In particular, any curve in $f\left(\mathbb{R}^{2}\right)$ must have infinite length, since otherwise its preimage would be a non-degenerate curve of zero length, a contradiction. Recall that a homeomorphism $f: X \rightarrow Y$ is called quasisymmetric if there is a homeomorphism $\eta:[0, \infty) \rightarrow[0, \infty)$ such that

$$
|x-y| \leq t|x-z| \quad \text { implies } \quad|f(x)-f(y)| \leq \eta(t)|f(x)-f(z)| .
$$

Quasiconformal maps of $\mathbb{R}^{d}, d \geq 2$, to itself are quasisymmetric (e.g., [4]), so Theorem 1.1 answers a question of S. Rohde (Question 18 of [2]) by implying

Corollary 1.2. There is a quasisymmetric embedding of $\mathbb{R}^{2}$ into $\mathbb{R}^{3}$ such that the image $f\left(\mathbb{R}^{2}\right)$ contains no rectifiable curves.

Received by the editors September 22, 1997.

1991 Mathematics Subject Classification. Primary 30C65.

Key words and phrases. Quasisymmetric maps, quasiconformal mappings, rectifiable curves, Jacobian.

The author was supported in part by NSF grant \# DMS 95-00577.

(C)1999 American Mathematical Society 
In this case, one can avoid the theorem that quasiconformal implies quasisymmetric; our function $\varphi$ will be constructed so that $\varphi(t r) \leq \varphi(t) \varphi(r)$, and this combined with (1) easily implies $f$ is quasisymmetric on $\mathbb{R}^{2}$.

In [3], J. Heinonen and P. Koskela define, for a quasiconformal map $f$ on the upper half space $\mathbb{R}_{+}^{n}$,

$$
a_{f}(x)=\exp \left\{n^{-1} \operatorname{vol}(B(x, r))^{-1} \int_{B(x, r)} \log J_{f}(y) d y\right\}
$$

( $J_{f}$ denotes the Jacobian of $f$ and $r$ is half the distance of $x=\left(x_{1}, \ldots, x_{n}\right)$ to the boundary of the half space), and

$$
E_{\infty}=\left\{x \in \partial \mathbb{R}_{+}^{n}: 0<\liminf _{r \rightarrow 0} a_{f}\left(x+r e_{n}\right) \leq \limsup _{r \rightarrow 0} a_{f}\left(x+r e_{n}\right)=\infty\right\} .
$$

Question 6 of [3] asks if there is an example where $E_{\infty}$ has positive Lebesgue measure and whether it can be the whole boundary. In our example, the Jacobian at $(x, y, t)$ is comparable to $(\varphi(t) / t)^{3}$, and thus $E_{\infty}$ is the whole boundary.

It is unknown whether there is an embedding of $\mathbb{R}^{2}$ into $\mathbb{R}^{3}$ which satisfies (1) with $\varphi(t)=t^{\alpha}$, for some $\alpha<1$ (it is easy to see that $\alpha<2 / 3$ is impossible). Such maps are easy to find taking $\mathbb{R}$ into $\mathbb{R}^{2}$ (just take the usual parameterization of the von Koch snowflake), and are known to exist for $\mathbb{R}^{d} \rightarrow \mathbb{R}^{n}$ if $n$ is large enough with respect to $d$ (see [1]).

After seeing my first proof of Corollary 1.2, S. Rohde pointed out the map I constructed on $\mathbb{R}^{2}$ extended to a quasiconformal map of $\mathbb{R}^{3}$ and hence gave Theorem 1.1. J. Heinonen then observed that this map solved the problem from [3]. I thank them for their comments and I thank the referees for numerous suggestions which improved the clarity of the paper.

\section{The PICTURE OF THE NON-RECTIFIABLE SURFACE}

We will start by describing a picture of the surface $f\left(\mathbb{R}^{2}\right)$ which gives the main idea of the construction. We will then give a more careful construction of the map on all of $\mathbb{R}^{3}$.

Let $\psi: \mathbb{R} \rightarrow \mathbb{R}$ be a smooth 1-periodic function with $\psi(0)=0$ and such that the length of the graph $\Gamma$ of $\psi$ above $[0,1]$ is 2 . Define a surface $S \subset \mathbb{R}^{3}$ by $S=\Gamma \times[0,1]$. Then $S$ is a smooth surface of area 2 and is isometric to $[0,2] \times[0,1]$ (with respect to the intrinsic path metric).

Start with the unit square $Q_{0}$ in $\mathbb{R}^{2}$. Define a map $f_{0}: Q_{0} \rightarrow \mathbb{R}^{3}$ by first stretching the square in the $x$ direction by a factor of 2 (i.e., apply the map $(x, y) \rightarrow$ $(2 x, y)$ and then map the image rectangle isometrically onto the surface $S$; see Figure 1).

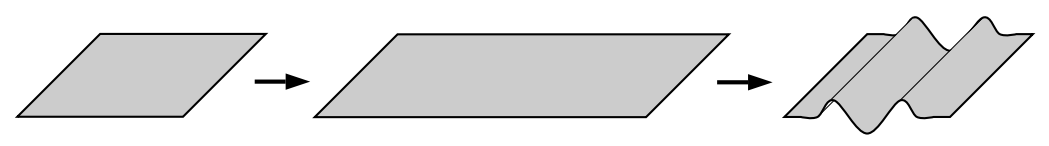

Figure 1. The map $F_{1}$ : Stretching a square and folding it.

Now choose a large integer $k_{1}$. The dyadic subsquares of $Q_{0}$ of size $2^{-k_{1}}$ are mapped by $F_{1}$ to pieces of $S$ which look like small rectangles of size $2^{-k_{1}+1} \times 2^{-k_{1}}$, and since $F_{2}$ is smooth, the approximation is better and better the larger $k_{1}$ is. 
Replace each such approximate rectangle by a scaled copy of $[0,1] \times \Gamma$, now oriented to the "oscillations" of the surface are orthogonal to the first set. See Figure 2. The scaled copies will not quite meet along their edges, but we can make them do so by bending them with arbitrarily small angle (depending on $k_{1}$ ). Because we have stretched in the $y$ direction, this time we see that small enough squares of size $r$ in $Q_{0}$ are now mapped onto regions which approximate small squares of size $2 r \times 2 r$, within a small error.

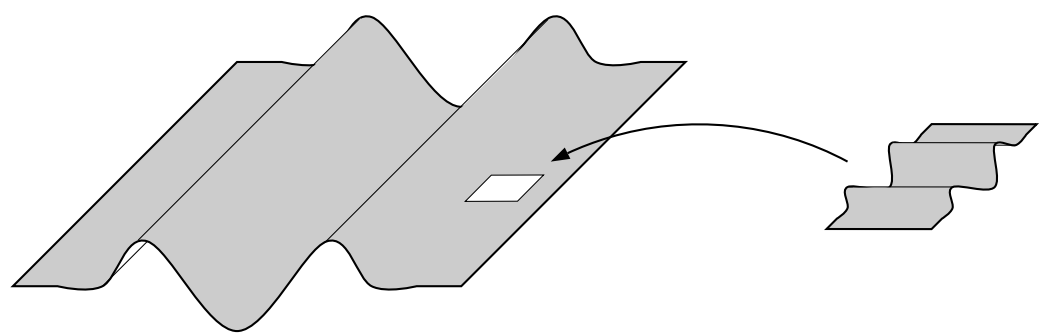

FiguRE 2. Folding in the orthogonal direction

We now continue by induction. After $n$ steps, we have a smooth surface $f_{n}\left(Q_{0}\right)$ which maps small squares of size $r=2^{-k_{n}}$ to pieces of the surface which approximate a $2^{n} r \times 2^{n} r$ square with small error. Next take each dyadic square in $Q_{0}$ of some size $\ll r$ and stretch by a factor of 2 in the $x$ direction and map it onto a scaled copy of $S$, bending it to make the edges fit together. Then stretch in the $y$ direction on an even smaller scale. If we choose the $\left\{k_{n}\right\}$ to increase quickly enough, then (1) will hold for $\varphi(t)=t 2^{\alpha(t)}$, where $\alpha(t)$ is the minimal $n$ so $2^{-k_{n}}<t$.

\section{The Construction}

Now we give a more careful construction of the desired map on $\mathbb{R}^{3}$. It will be the limit of a sequence of compositions $f_{n}=g_{1} \circ \cdots \circ g_{n}$ where the $g_{i}$ 's are given by the following

Lemma 3.1. There is a $C<\infty$, so that for any $0<\delta, \epsilon, \eta \leq 1$, there is a $\nu=$ $2^{-m}>0$ and a quasiconformal map $g: \mathbb{R}^{3} \rightarrow \mathbb{R}^{3}$ so that if $z=(x, y, t) \in \mathbb{R}^{3}$, then

1. $g(z)=z$ if $|t|>\delta$.

2. The linear dilatation of $g$ is $\leq 1+C(\epsilon)$ in all of $\mathbb{R}^{3}$, where $C(\epsilon) \rightarrow 0$ as $\epsilon \rightarrow 0$.

3. The linear dilatation of $g$ is $\leq 1+\eta$ if $|t|<\nu$.

4. For $z, w \in \mathbb{R}^{2} \times\{0\}$, we have

$$
\left.\begin{array}{rl}
(1+\epsilon)(1-\eta) \\
1-\eta \\
\left(1+\frac{C \delta}{|z-w|}\right)^{-1}
\end{array}\right\} \leq \frac{|g(z)-g(w)|}{|z-w|} \leq \begin{cases}1+\epsilon+\eta, & |z-w| \leq \nu . \\
1+\epsilon+\eta, & \nu \leq|z-w| \leq \delta . \\
1+\frac{C \delta}{|z-w|}, & |z-w| \geq \delta .\end{cases}
$$

First we check that Theorem 1.1 follows from the lemma. Choose a sequence $\left\{\epsilon_{n}\right\}$ in $(0,1]$, so that $\sum_{n} \epsilon_{n}=\infty$. For example, $\epsilon_{n}=1$ for all $n$ will correspond to the picture described in the previous section and taking $\epsilon_{n} \rightarrow 0$, will give an asymptotically flat example. Let $\eta_{n}=10^{-n}$. Let $\delta_{1}=1$. Now apply the lemma with $\epsilon_{1}, \delta_{1}, \eta_{1}$ to obtain a quasiconformal mapping $g_{1}$, and a positive number $\nu_{1}$. In general, given $\nu_{n-1}$, choose $\delta_{n}<\min \left(\nu_{n-1} / 8, \delta_{n-1} / 100\right)$, and apply the lemma to the triple $\epsilon_{n}, \delta_{n}, \eta_{n}$, to obtain the map $g_{n}$ and the number $\nu_{n}$. Let $f_{n}=g_{1} \circ \cdots \circ g_{n}$. 
Then $f_{n}$ is a finite composition of quasiconformal maps and hence is quasiconformal. It is the identity map on $\{z=(x, y, t):|t| \geq 1\}$ and to estimate its dilatation at a point $z$ with $|t|<1$, choose $k$ so that $\delta_{k+1} \leq|t|<\delta_{k}$. Then $g_{k+1} \circ \cdots \circ g_{n}$ is the identity at $z$. The map $g_{k}$ has linear dilatation at most $1+C\left(\epsilon_{k}\right)$ everywhere, and hence at $z$. For each $1 \leq j<k$, the image of $z$ under $g_{j} \circ \cdots \circ g_{k}$ is in $\left\{z=(x, y, t):|t|<\delta_{j}\right\}$ (since the inverse is the identity on on the complement) and hence the dilatation is at most $1+\eta_{j}$. Thus the linear dilatation of $f_{n}$ at $z$ is at most $\left(1+\sup _{k \leq n} C\left(\epsilon_{k}\right)\right) \prod_{k \leq n}\left(1+\eta_{j}\right) \leq C$. It is easy to check that $\left\{f_{n}\right\}$ is Cauchy with respect to uniform convergence on compacta, so $f=\lim _{n} f_{n}$ exists and is quasiconformal.

To prove (1) choose $z, w \in \mathbb{R}^{2}$ and choose $k$ so that $2 \delta_{k+1} \leq|z-w| \leq 2 \delta_{k}$. For $j=n-1, \ldots, 1$, let $z_{j}=g_{j} \circ \cdots \circ g_{n}(z)$ and similarly for $w_{j}$. For $k<j \leq n-1$, induction and the fact that $\prod\left(1-\left(\delta_{j} / \delta_{k+1}\right)\right)>1 / 2$ imply

$$
\left|z_{j}-w_{j}\right| \geq\left(1-\eta_{j}\right)\left|z_{j+1}-w_{j+1}\right| \geq \frac{1}{2}|z-w| \geq \delta_{k+1} \geq \delta_{j}
$$

Thus case 3 of part 4 of the lemma holds and

$$
\left(1+C \frac{\delta_{j}}{\delta_{k+1}}\right)^{-1} \leq \frac{\left|z_{j-1}-w_{j-1}\right|}{\left|z_{j}-w_{j}\right|} \leq\left(1+C \frac{\delta_{j}}{\delta_{k+1}}\right) .
$$

For $j=k$ we have (no matter which case holds),

$$
\left(1-\eta_{k}\right)\left|z_{k+1}-w_{k+1}\right| \leq\left|z_{k}-w_{k}\right| \leq 2\left(1+\eta_{k}\right)\left|z_{k+1}-w_{k+1}\right| .
$$

For $j=k-1,\left|z_{k-1}-w_{k-1}\right| \leq 4|z-w| \leq 8 \delta_{k} \leq \nu_{k-1}$ so case 1 holds in part 4 of the lemma. Furthermore, for $j=k-2, \ldots, 1,\left|z_{j-1}-w_{j-1}\right| \leq 4\left|z_{j}-w_{j}\right|$ and $\nu_{j}<\delta_{j} \leq \nu_{j} / 4$. Thus $\left|z_{j}-w_{j}\right| \leq \nu_{j}$ implies $\left|z_{j-1}-w_{j-1}\right| \leq \nu_{j-1}$. Thus for $j<k$, case 1 of part 4 of Lemma 3.1 applies, and so

$$
1-\eta_{j} \leq \frac{\left|z_{j-1}-w_{j-1}\right|}{\left(1+\epsilon_{j}\right)\left|z_{j}-w_{j}\right|} \leq 1+\eta_{j}
$$

Putting all these together and telescoping we get,

$$
\begin{aligned}
\prod_{j=k+1}^{n}\left(1+C \frac{\delta_{j}}{\delta_{k+1}}\right)^{-1} \prod_{j=1}^{k}\left(1-\eta_{j}\right) \leq & \frac{\left|z_{1}-w_{1}\right|}{|z-w| \prod_{j=1}^{k}\left(1+\epsilon_{j}\right)} \\
& \leq 2 \prod_{j=k+1}^{n}\left(1+C \frac{\delta_{j}}{\delta_{k+1}}\right) \prod_{j=1}^{k}\left(1+\eta_{j}\right) .
\end{aligned}
$$

Since the products converge (independent of $k$ and $n$ ) and $f(z)=z_{1}, f(w)=w_{1}$, we have

$$
C^{-1} \leq \frac{|f(z)-f(w)|}{\varphi(|z-w|)} \leq C
$$

where $\varphi(t)=t \prod_{j=1}^{\alpha(t)}\left(1+\epsilon_{j}\right)$, with $\alpha(t)$ defined by $\alpha(t)=\max \left\{k: t \leq 2 \delta_{k}\right\}$. This is (1). Since $\sum \epsilon_{n}=\infty$, we also have (2).

Thus Lemma 3.1 proves Theorem 1.1. Now we prove the lemma. We will build $g$ as a composition $g=g_{1} \circ g_{2} \circ g_{3}$. Let $\psi: \mathbb{R} \rightarrow \mathbb{R}$ be a smooth 1-periodic function with $\psi(0)=0$ and such that the length of the graph $\Gamma$ of $\psi$ above $[0,1]$ is $1+\epsilon$. Define a surface $S \subset \mathbb{R}^{3}$ by $S=\Gamma \times \mathbb{R}$. Let $L(x)=\int_{0}^{x} \sqrt{1+\left|\psi^{\prime}(t)\right|^{2}} d t$ be the 
arclength of the graph over $[0, x]$ and let $J$ be the inverse, $L^{-1}$. Define a map $F: \mathbb{R}^{2} \rightarrow S \subset \mathbb{R}^{3}$ by

$$
F:(x, y) \rightarrow(J((1+\epsilon) x), y, \psi(J((1+\epsilon) x))) .
$$

Now extend $F$ to a function $G$ defined on all of $\mathbb{R}^{3}$ by setting $G(x, y, t)=(x, y, t)$ if $|t| \geq 1, G$ is quasiconformal with dilatation at most $1+C(\epsilon)$, and the dilatation of $G$ tends to 1 as $t \rightarrow 0$. For example, we can do this by making sure that $\partial_{t} G(x, y, 0)$ has norm 1 and is perpendicular to $S=F\left(\mathbb{R}^{2}\right)$, e.g., set

$$
G(x, y, t)=F(x, y)+t \cdot N(x, y)
$$

for small enough $t<t_{0}$, where

$$
N(x, y)=\frac{\left(-\psi^{\prime}(J((1+\epsilon) x), 0,1)\right.}{\sqrt{\left|\psi^{\prime}(J((1+\epsilon) x))\right|^{2}+1}},
$$

is the normal vector to $F\left(\mathbb{R}^{2}\right)$ at the point $F(x, y)$.

To define $g_{1}$, let $g_{1}(x, y, t)=\frac{\delta}{2} G\left(\frac{2}{\delta}(x, y, t)\right)$. Thus the tangent map near $\mathbb{R}^{2} \times\{0\}$ looks like an affine stretch by a factor of $1+\epsilon$ in the $x$ direction, followed by a rotation in the $(x, t)$ plane.

To define the map $g_{2}$ we do a similar thing, but at a much smaller scale and reversing the roles of $x$ and $y$. Choose $\nu_{1}$ so small that $g_{1}$, restricted to the set $\left\{(x, 0, t): t<\nu_{1}\right\}$, has linear dilatation $\leq 1+\eta / 10$. Now let $g_{2}(x, y, t)=$ $\nu_{1} G\left(\nu_{1}^{-1}(y, x, t)\right)$. Choose $\nu_{2}$ so that $g_{2}$ has linear dilatation $\leq 1+\eta / 10$ on $\{(0, y, t)$ : $\left.t<\nu_{2}\right\}$. Thus near $\mathbb{R}^{2}$, the tangent map looks like an affine stretch in the $y$ direction, followed by a rotation in the $(y, t)$ plane.

Finally, define $g_{3}$ by

$$
g_{3}(x, y, t)= \begin{cases}(x, y, t), & \text { if } \quad|t| \geq \nu_{2} / 2, \\ \left(x, y, \nu_{2} h\left(t / \nu_{2}\right)\right), & \text { if } \quad \nu_{2} / 8 \leq|t| \leq \nu_{2} / 2, \\ (x, y,(1+\epsilon) t), & \text { if } \quad t \geq \nu_{2} / 8\end{cases}
$$

where $h$ smoothly interpolates between $(1+\epsilon) / 8$ and $1 / 2$ on $\left[\frac{1}{8}, \frac{1}{2}\right]$ and satisfies $\left|h^{\prime}-1\right| \leq C(\epsilon)$. Thus near $\mathbb{R}^{2}$, the map is an affine stretch in the $t$ direction and $g_{3}$ is $1+C(\epsilon)$ quasiconformal.

We claim that $g=g_{1} \circ g_{2} \circ g_{3}$ satisfies the lemma with $\nu=\nu_{2} / 16$. Most of the conditions are clear from the construction. For example, it is clear that $g$ is quasiconformal and is the identity on $\{(x, y, t):|t|>\delta\}$. Moreover, it has dilatation bounded everywhere by $1+C(\epsilon)$ (since each of the three component maps does). Finally, for points in $\{(x, y, t):|t|<\nu\}$, by considering the tangent maps of the composition, it is easy to see that the map acts as a conformal dilation of size $1+\epsilon$ with error at most $\eta$. This also proves the expansion estimates on the small and intermediate scales (i.e., $|z-w| \leq \delta$ ). For large scales (i.e., $|z-w| \geq \delta$ ), we just use the fact that $|G(z)-G(w)|=|z-w|+O(1)$ which implies $|g(z)-g(w)|=$ $|z-w|+O(\delta)$. This completes the proof of the lemma.

Added in proof. G. David and T. Toro have improved Corollary 1.2 by showing that there is an embedding of $\mathbb{R}^{2}$ into $\mathbb{R}^{3}$ which satisfies (1) with $\varphi(t)=t^{\alpha}$ for some $\alpha<1$. Their result appears in a preprint "Reifenberg flat metric spaces, snowballs and embeddings". 


\section{REFERENCES}

[1] Assouad, P. (1983) Prolongements Lipschitziens dans $\mathbb{R}^{n}$, Bull. Soc. Math. France 111, 429-448. MR 86f:54050

[2] Heinonen, J. and Semmes, S. (1997) Thirty-three yes or no questions about mappings, measures and metrics, Conf. Geometry and Dynamics 1, 1-12. CMP 97:13

[3] Heinonen, J. and Koskela, P. (1994) The boundary distortion of a quasiconformal mapping, Pacific J. Math 165, 93-114. MR 95f:30031

[4] P. Tukia and J. Väisälä (1980) Quasisymmetric embeddings of metric spaces. Ann. Acad. Sci. Fenn. Ser. A I Math. 5, 97-114. MR 82g:30038

Department of Mathematics, State University of New York at Stony Brook, Stony Brook, New York 11794-3651

E-mail address: bishop@math.sunysb.edu 